\title{
EVALUACIÓN DEL NIVEL CONTAMINANTE DE OCRATOXINA A (OTA) MEDIANTE COLUMNAS DE INMUNOAFINIDAD Y CROMATOGRAFÍA LÍQUIDA DE ALTA EFICIENCIA EN Coffea arabica L. "CAFE'"
}

Félix Hugo Milla Flores ${ }^{* a}$, Américo Castro Luna ${ }^{a}$.

\begin{abstract}
RESUMEN
El estudio tiene como objetivo evaluar el nivel contaminante de la ocratoxina A (OTA) en Coffea arabica L. "café", de tipo tostado, purificado mediante columnas de inmunoafinidad y cuantificado por Cromatografía Líquida de Alta Eficiencia (HPLC). La OTA es una micotoxina nefrotóxica-cancerígena capaz de causar efectos adversos sobre la salud humana y animal. Se encuentra en muchos alimentos procesados y no procesados, entre ellos el café, que es un alimento de elevado consumo. La recolección de muestras de café tostado se realizó en la zona cafetalera de Chanchamayo, departamento de Junín-Perú. La separación y purificación de la ocratoxina A se realizó en columnas de inmunoafinidad NeoColumn, posteriormente su cuantificación se realizó por HPLC con detector de fluorescencia a una longitud de onda de excitación de $333 \mathrm{~nm}$ y longitud de onda de emisión de $460 \mathrm{~nm}$. Finalmente, se realizaron pruebas de recuperación a un nivel contaminante de $5 \mathrm{ppb}$. Los resultados obtenidos en las muestras de café tostado presentan un contenido de 0,216 y 0,444 ppb de OTA los cuales no superan los límites permitidos por la legislación internacional. El porcentaje de recuperación al nivel de $5 \mathrm{ppb}$ fue de 76,231\%. El estudio remarca la importancia de la trazabilidad en un producto de consumo frecuente a nivel nacional e internacional con la finalidad de garantizar su inocuidad.
\end{abstract}

Palabras clave: Micotoxina, ocratoxina A, café tostado, columna de inmunoafinidad, cromatografía líquida de alta afinidad.

a Facultad de Farmacia y Bioquímica, Universidad Nacional Mayor de San Marcos, Jr Puno 1002. Lima- Perú. fmillaf@gmail.com 


\title{
EVALUATION OF THE POLLUTANT LEVEL OF OCHRATOXIN A (OTA) THROUGH IMMUNOAFFINITY COLUMNS AND HIGH EFFICIENCY LIQUID CHROMATOGRAPHY IN Coffea arabica L. "COFFEE"
}

\begin{abstract}
The study aims to evaluate the contaminant level of Ochratoxin A (OTA) in Coffea arabica L. "coffee", roasted, purified by immunoaffinity columns and quantified by High Efficiency Liquid Chromatography (HPLC). OTA is a nephrotoxic-carcinogenic mycotoxin capable of causing adverse effects on human and animal health. It is found in many processed and unprocessed foods, including coffee, which is a food with high consumption. Roasted coffee samples were collected in the coffee zone of Chanchamayo, department of Junin-Peru. The separation and purification of the Ochratoxin A was performed by NeoColumn immunoaffinity columns, subsequently quantification was performed by HPLC with fluorescence detector, at an excitation wavelength of $333 \mathrm{~nm}$ and emission wavelength of $460 \mathrm{~nm}$. Finally, the test of percent of recovery were carried out at the contaminant level of $5 \mathrm{ppb}$. The results obtained in roasted coffee samples have a content of 0,216 and 0,444 ppb of OTA which do not exceed the limits allowed by international law. The recovery rate at level $5 \mathrm{ppb}$ was 76,231\%. The study highlights the importance of traceability in a product of frequent consumption at national and international level, in order to ensure its safety.
\end{abstract}

Key words: Mycotoxin, ochratoxin A, roasted coffee, immunoaffinity column, high affinity liquid chromatography.

\section{INTRODUCCIÓN}

La ocratoxina A es comúnmente producida por los mohos Aspergillus ochraceus y Penicillium viridicatum; su presencia está asociada a los productos como el maíz, la cebada, el café verde y varias frutas secas ${ }^{1}$. La ocratoxina A puede estar presente en conjunción con las aflatoxinas, sustancias consideradas como carcinógenos naturales. La ocratoxina A es un potencial agente carcinógeno, que afecta los riñones de los animales expuestos a dicha micotoxina ${ }^{2}$.

Se estima que una cuarta parte de las cosechas mundiales están contaminadas por micotoxinas. Un estudio analizó 2753 muestras de cereales y piensos, revelando que más de la mitad de las muestras de origen europeo y la tercera parte de la región Asia - Pacífico estaban contaminadas con niveles de micotoxinas por encima del límite de cuantificación ${ }^{3}$.

El consumo mundial de café supera los seis millones de toneladas al año y la venta minorista, principalmente en Europa, EE.UU. y el Japón, se encuentra alrededor de los 70000 millones de dólares al año. Los países en desarrollo que producen la materia prima reciben unos 6000 millones de dólares ${ }^{4}$. 
Aunque no hay un nivel consultivo o regulatorio para la ocratoxina A emitido por la Administración de Alimentos de los EE.UU., muchos coinciden que los niveles entre 1020 partes por mil millones (ppb) en productos destinados al consumo humano o animal pueden causar problemas de salud y pérdidas económicas. Algunos mercados extranjeros han establecido límites de regulación que van desde 5-50 ppb para el comercio interno, mientras que la Comisión Europea desde el año 2006 ha establecido un límite máximo de 5 ppb para café tostado.

La mejor protección contra las micotoxinas es controlar su presencia en piensos y alimentos. Eso significa mantener un control en todo el camino productivo desde la cosecha inicial de granos hasta el producto terminado.

\section{PARTE EXPERIMENTAL}

El estudio se realizó en el Instituto de Investigación en Ciencias Farmacéuticas y Recursos Naturales "Juan de Dios Guevara" de la Facultad de Farmacia y Bioquímica de la Universidad Nacional Mayor de San Marcos y en el Laboratorio de Investigación y Certificaciones (LABICER) de la Facultad de Ciencias de la Universidad Nacional de Ingeniería.

\section{Reactivos}

Acetonitrilo de grado HPLC de la marca J.T Baker, estándar de ocratoxina A (OTA) 99,18 \%, metanol grado HPLC (Merck), ácido acético $\left(\mathrm{CH}_{3} \mathrm{COOH}\right)$ grado analítico $100 \%$, Buffer fosfato salino en tabletas $\mathrm{pH}=7,40$ (PBS) y Tween 20 adquiridos de la casa comercial Merck. El agua utilizada fue purificada en un equipo Elga Purelab CLASSIC UV.

\section{Equipos}

Cromatógrafo Líquido de Alto Rendimiento-SHIMADZU que posee: desgasificador DGU20A5R, automuestreador SIL-30AC, bomba cuaternaria LC-30AD, horno de columna: CTO-20AC, detector de fluorescencia RF-20Axs, control de sistema CBM-20A; Columna cromatográfica RP C18 de $250 \mathrm{~mm}$ x 4,6 mm ID x $5 \mu \mathrm{m}$ partícula (Restek); Molinillo Bosch, agitadores magnéticos de las marcas Thermo Scientific Cimarec e IKA C-MAG HS7; Balanza analítica digital GR-300 AND, equipo ultrasonido WISD Laboratory Instruments; Ultrapurificador de agua ELGA PURELAB CLASSIC UV y columnas de inmunoafinidad para ocratoxina NEOCOLUMN.

\section{Colecta de la muestra}

Los granos de café tostado provinieron del departamento de Junín, provincia de Chanchamayo, distrito Perené del anexo Pampa Tigre. Las actividades de muestreo se realizaron a temperatura ambiente y humedad atmosférica característica de la zona en el mes de noviembre de 2018. Las muestras fueron rotuladas y transportadas al departamento de Lima para su tratamiento y respectivos análisis. 


\section{Tratamiento de la muestra}

Los granos de café fueron secados en una estufa a $38^{\circ} \mathrm{C}$ por 24 horas. Las muestras de café tostado se molieron en un molinillo Bosch. Una vez trituradas se homogenizaron y se conservaron a temperatura de refrigeración en el rango de $0^{\circ} \mathrm{C}$ a $4^{\circ} \mathrm{C}$ para su posterior análisis.

\section{Preparación de los estándares de ocratoxina A}

La solución principal del estándar de OTA se preparó diluyendo $1 \mathrm{mg}$ de estándar OTA en $50 \mathrm{~mL}$ de mMetanol, teniendo una concentración de $20 \mathrm{ppm}$. A partir de la solución principal, se preparó la solución de trabajo a una concentración de 200 ppb y a partir de esta solución se prepararon los diferentes estándares utilizados para la construcción de la curva de calibración, cuyas concentraciones variaron de 0,05 a $10 \mathrm{ppb}$. De la solución madre se prepararon las soluciones utilizadas en las pruebas de recuperación del método utilizado en granos tostados.

\section{Extracción y purificación de la ocratoxina A en las muestras}

Se utilizó $10 \mathrm{~g}$ de café tostado finamente molido, y pasado por un tamiz de $0,5 \mathrm{~mm}$. Para las extracciones se utilizó $100 \mathrm{ml}$ de metanol con bicarbonato en una proporción de 7:3 de metanol y solución de bicarbonato de sodio al $1 \%$, respectivamente. Luego, se mezcló en un agitador magnético a alta velocidad durante tres minutos, se dejó en reposo por tres minutos y se filtró el sobrenadante líquido con un papel Whatman $\mathrm{N}^{\circ} 426-7$.

Se mide cinco mililitros del filtrado y se diluye con $45 \mathrm{ml}$ de PBS / Tween $(0,01 \% \mathrm{v} / \mathrm{v})$ obteniendo un volumen final de $50 \mathrm{ml}$. Con una de jeringa de $50 \mathrm{ml}$ y un adaptador de columna, se eluyó la muestra de $50 \mathrm{ml}$ a través de la columna de inmunoafinidad gota a gota. Se tomó en consideración que la columna no se seque. Posteriormente, se lavó la columna con $20 \mathrm{ml}$ de PBS / Tween $(0,01 \% \mathrm{v} / \mathrm{v})$ y con $10 \mathrm{ml}$ de PBS, manteniendo las mismas consideraciones. Se aseguró en eliminar todo el líquido de la columna utilizando presión positiva de una jeringa ${ }^{8}$. La OTA se reconstitutyó con $0,75 \mathrm{~mL}$ de metanol acidificado; 0,75 $\mathrm{mL}$ de metanol y 1,5 de agua ultrapura, según las indicaciones del fabricante de Columnas de Inmunoafinidad Neocolumn.

\section{Cuantificación por cromatografía líquida de alto rendimiento}

Los análisis se realizaron mediante cromatografía líquida de alto rendimiento con detección por fluorescencia, la metodología y las condiciones del equipo propuestas por Tafuri ${ }^{9}$.

La longitud de onda de excitación fue de $333 \mathrm{~nm}$ y la longitud de onda de emisión fue de 460 $\mathrm{nm}$. La temperatura del horno de la columna fue de $25^{\circ} \mathrm{C}$. La elución fue a un flujo constante de $1 \mathrm{~mL} / \mathrm{min}$ de $\mathrm{CH}_{3} \mathrm{CN}$ (ácido acético $1 \%$ ) y $\mathrm{H}_{2} \mathrm{O}$ (ácido acético $1 \%$ ) (50:50 v/v) como el sistema inicial de eluyentes. La gradiente de elución, se inició con el acondicionmiento del sistema de solventes por 20 min desde $50 \%$ hasta $100 \%$ de $\mathrm{CH}_{3} \mathrm{CN}$ la cual se mantuvo durante 3 minutos; los últimos 10 minutos el sistema se restableció a sus condiciones iniciales de $\mathrm{CH}_{3} \mathrm{CN} 50 \%$ y $\mathrm{H}_{2} \mathrm{O} 50 \%$. 
Los eluyentes y las muestras fueron filtrados a través de filtro de jeringa $(0,22 \mu \mathrm{m})$, el volumen de inyección a la columna cromatográfica fue de $25 \mu \mathrm{L}$.

Las lecturas de los estándares de OTA y muestras problemas se realizaron por duplicado. La identificación de OTA se realizó utilizando el tiempo promedio de retención de las diluciones de los estandares. Este tiempo de retención y Límite de Detección (LDD) se determinó midiendo diez veces el estándar de trabajo a una concentración de 0,09918 ppb, El tiempo de retención promedio fue de 7,964 $\pm 0,010$ minutos y el LDD promedio fue de $0,019 \pm 0.001$ ppb.

\section{Pruebas de recuperación del método utilizado}

Para determinar la eficacia en el proceso de recuperado se procedió elaborando un blanco para la muestra de granos de café tostado, adicionado a una concentracion de 5 ppb con estándar de OTA. Las muestras adicionadas se trataron de igual manera que las muestras problemas.

\section{RESULTADOS Y DISCUSIÓN}

La identificación de la ocratoxina A se realizó utilizando el tiempo de retención promedio de las diluciones de trabajo, el cual fue de 7,964 min, a partir del estándar de menor concentración $(0,09918 \mu \mathrm{g} / \mathrm{L})$ se realizaron 10 repeticiones.

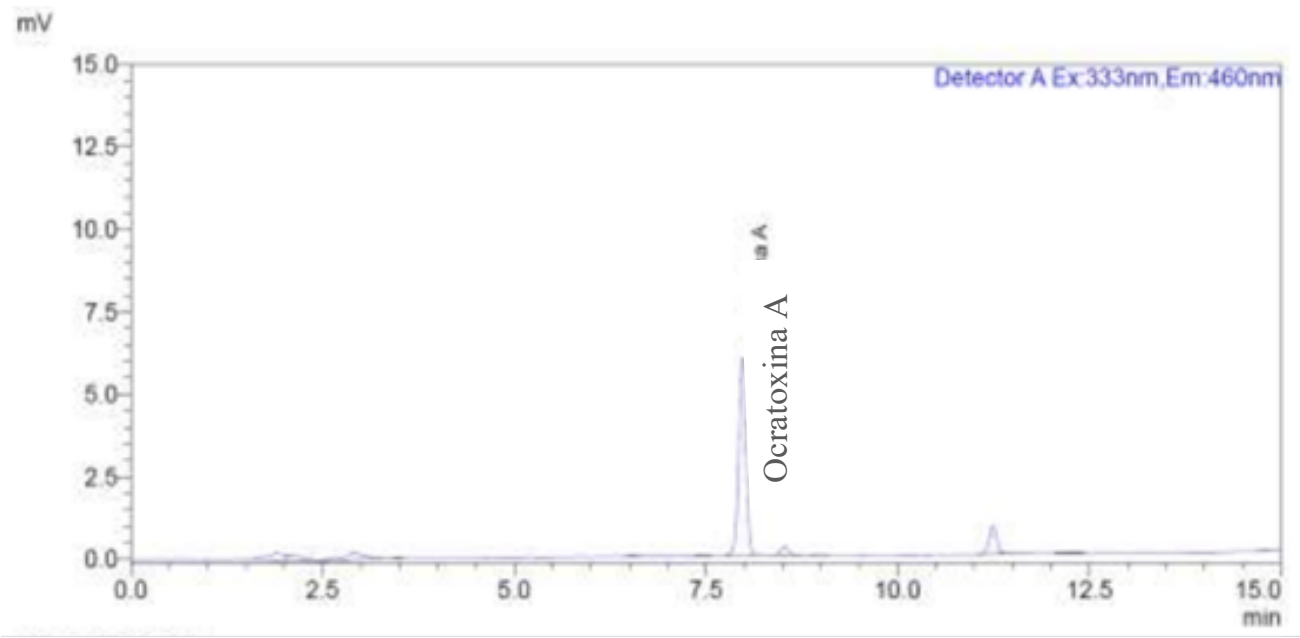

Figura 1. Cromatograma del estándar de ocratoxina A a concentración de 9,632. 


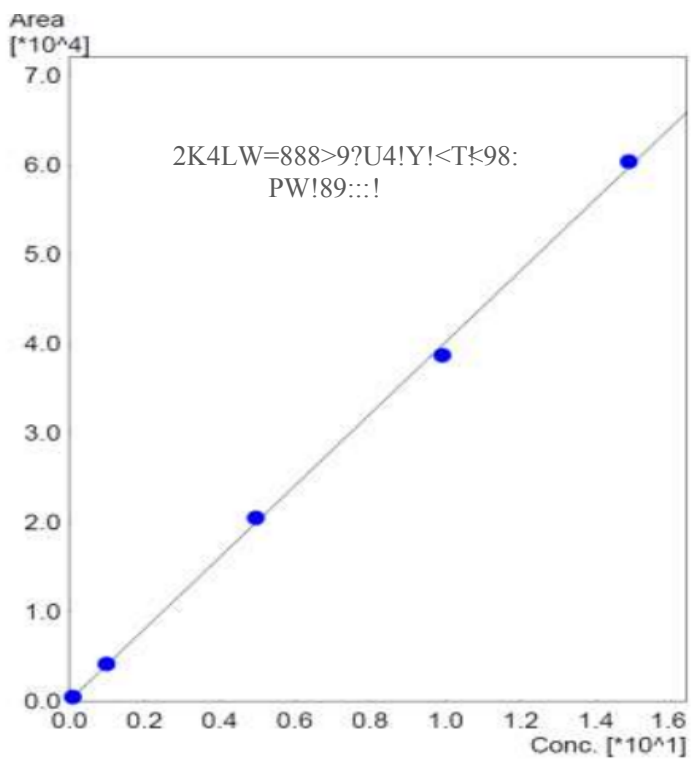

Figura 2. Curva de calibración utilizada para la cuantificación de ocratoxina A a concentraciones promedios de 0,$099 ; 0,991 ; 4,959 ; 9,918 ; 14,877 \mu \mathrm{g} / \mathrm{L}$.

El método de recuperación utilizado para el análisis de OTA fue evaluado al nivel de 5 ppb (a) de OTA, los resultados se muestran en la tabla $1 .^{5}$

Tabla 1. Contenido de OTA en las muestras adicionadas de café tostado.

\begin{tabular}{cccccc}
\hline Muestra & $\begin{array}{c}\text { Tiempo de } \\
\text { retención }\end{array}$ & Área & Altura & Concentración & \%RSD* \\
\hline Café tostado & 7,982 & 2697 & 416 & $0,643 \mu \mathrm{g} / \mathrm{L}$ & 1,760 \\
\hline
\end{tabular}

$* \mathrm{RSD}=$ desviacion estandar relativa. El porcentaje de recuperacion fue de $76,231 \%$ evaluado al nivel de $5 \mathrm{ppb}$. 


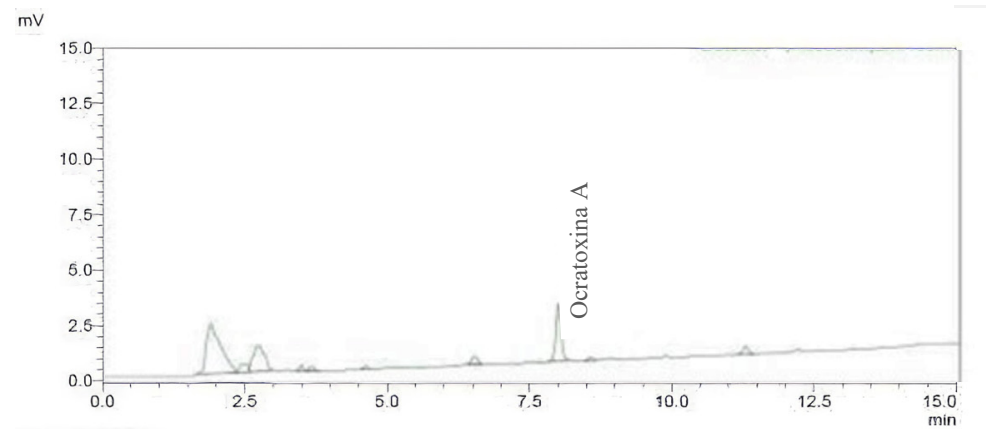

Figura 3. Cromatograma de la prueba de recuperación de la muestra con adicion de $5 \mathrm{ppb}$ de OTA.

El propósito de la extracción es fijar la ocratoxina A en la matriz de análisis, es decir sobre el material de la columna de inmunoafinidad, usando solventes adecuados para su posterior recuperación y cuantificación. Los solventes de extracción y el método utilizado son las dos consideraciones más importantes para el procedimiento de extracción de ocratoxina $\mathrm{A}^{10}$. La selección del solvente de extracción depende de varios factores, incluidas las características físicas y químicas del analito, el costo y la seguridad del solvente, la solubilidad de compuestos diferentes a la ocratoxina A (interferencia por colorantes u otros componentes) y los procesos después de la extracción ${ }^{11}$.

En la investigación se utilizó columnas de inmunoafinidad para ocratoxina A de la marca NeoColumn código del producto 8640 , fabricadas por NeoGen ${ }^{\circledR}$ Corporation, el cual posee un método de extracción validado para muestras de café verde, tostado e instantáneo, aunque existe diversos variantes de los sistemas de solventes de extracción de OTA en cafée ${ }^{9-12}$.

Se recomienda el uso de una disolución de metanol al $60 \%$ y agua al $40 \%{ }^{12}$. También, se han utilizado como solvente de extracción metanol y solución de bicarbonato de sodio al $3 \%$ en la relación $1: 1^{13}$. Al saber que la OTA es una molécula de polaridad media, se busca el uso de solventes polares y semipolares, esto es contrastable en diversas investigaciones, aunque suelen existir modificaciones para permitir mayores eficiencias en la extracción relacionadas a menos efectos de interferencia. Existen reportes utilizando solventes como la acetona, acetato de etilo, cloroformo para la extracción de ocratoxina A en matrices complejas ${ }^{14-16}$, cabe acotar que el uso de solventes apolares como en acetato de etilo para la extracción de OTA, puede ser necesario bajo un procedimiento de desengrase adicional antes de la detección o purificación, adicionalmente del uso de solventes semipolares acidificados para su reconocimiento por $\mathrm{HPLC}^{14}$.

Las muestras adicionadas con OTA presentaron un porcentaje de recuperación de 76,23\% al nivel de $5 \mathrm{ppb}$. Los límites de recuperación comúnmente aceptados para un nivel de contaminación de OTA de 10 ppb están comprendidos entre 70 a $125 \%$ de recuperación ${ }^{17}$. 
Para la concentración evaluada a 5 ppb de OTA, el porcentaje de recuperación del método de la Comunidad Europea ${ }^{5}$, se encontró entre estos los límites. Sin embargo, existen métodos en donde el porcentaje de recuperación para concentraciones más bajas de OTA (5 ppb) fue inferior al $70 \%$ con el método validado por la Unión Europea (UE) “14132”15. La recuperación por el método de Pittet $^{18}$, el cual emplea solventes como metanol y bicarbonato de sodio al $3 \%$, difiere del método usado en los volúmenes de extracción y soluciones estabilizadas de lavado (PBS pH 7,4).

Las condiciones de los solventes que presenten un $\mathrm{pH}$ demasiado alto conducen a la formación de OTA de anillo abierto (OP-OA), que no es reconocido por los anticuerpos OTA en el procedimiento de purificación o limpieza. Estas circunstancias ya han sido reportadas durante el análisis de otras matrices contaminadas con OTA ${ }^{19,20}$.

La dilución realizada en nuestro método y el uso de PBS ajustado a 7,4 de pH, permite que se realice la conversión de OP-OTA en OTA antes del proceso de purificación por columnas de inmunoafinidad. Los valores de recuperación son relativamente constantes con un promedio del $70 \%$. Se recomienda que las recuperaciones inferiores al $60 \%$ pueden estar sujetas a investigaciones que conduzcan a una mejora en los métodos y solventes de extracción ${ }^{17}$. Manteniendo la línea de investigación en la búsqueda de eficacia de los métodos de recuperación.

Los resultados obtenidos de OTA en muestras de granos de café tostado se muestran en la tabla 2 .

Tabla 2. Contenido de OTA en las muestras de café tostado.

\begin{tabular}{cccccc}
\hline Muestra & $\begin{array}{c}\text { Tiempo de } \\
\text { retención }\end{array}$ & Área & Altura & Concentración & \%RSD \\
\hline Café tostado & 8,077 & 407 & 37 & $0,071 \mu \mathrm{g} / \mathrm{L}$ & 1,498 \\
M1 & 8,066 & 413 & 37 & $0,073 \mu \mathrm{g} / \mathrm{L}$ & \\
Café tostado & 8,071 & 704 & 74 & $0,142 \mu \mathrm{g} / \mathrm{L}$ & 2,353 \\
M2 & 8,070 & 723 & 75 & $0,150 \mu \mathrm{g} / \mathrm{L}$ & \\
\hline
\end{tabular}

Las muestras fueron analizadas por duplicado reportando valores de $0,072 \pm 0,001$ y $1,248 \pm$ $0,003 \mu \mathrm{g} / \mathrm{L}$ equivalentes a 0,216 y $0,444 \mathrm{ppb}$ de OTA. Los resultados no superan los límites máximos permitidos de 5 ppb OTA en café tostado y molido, establecidos por la Unión Europea. 
Tabla 3. Cálculo de Límite de Detección (LDD)

\begin{tabular}{ccccccc}
\hline Repetición & $\begin{array}{c}\text { Tiempo de } \\
\text { retención }\end{array}$ & Área & Altura & Concentración & Ruido & LLD* $^{*}$ \\
\hline 1 & 7,959 & 463 & 75 & $0,0853 \mu \mathrm{g} / \mathrm{L}$ & 5,28 & 0,020 \\
\hline 2 & 7,955 & 443 & 73 & $0,0804 \mu \mathrm{g} / \mathrm{L}$ & 5,40 & 0,020 \\
\hline 3 & 7,961 & 460 & 75 & $0,0845 \mu \mathrm{g} / \mathrm{L}$ & 5,35 & 0,020 \\
\hline 4 & 7,963 & 456 & 73 & $0,0837 \mu \mathrm{g} / \mathrm{L}$ & 5,22 & 0,020 \\
\hline 5 & 7,978 & 449 & 73 & $0,0819 \mu \mathrm{g} / \mathrm{L}$ & 4,81 & 0,018 \\
\hline 6 & 7,971 & 448 & 73 & $0,0817 \mu \mathrm{g} / \mathrm{L}$ & 5,50 & 0,020 \\
\hline 7 & 7,954 & 464 & 75 & $0,0857 \mu \mathrm{g} / \mathrm{L}$ & 5,10 & 0,019 \\
\hline 8 & 7,954 & 471 & 75 & $0,0872 \mu \mathrm{g} / \mathrm{L}$ & 5,01 & 0,020 \\
\hline 9 & 7,981 & 455 & 73 & $0,0834 \mu \mathrm{g} / \mathrm{L}$ & 4,60 & 0,017 \\
\hline 10 & 7,989 & 448 & 71 & $0,0816 \mu \mathrm{g} / \mathrm{L}$ & 5,12 & 0,019 \\
\hline Promedio & 7,964 & 456,6 & 73,9 & $0,0838 \mu \mathrm{g} / \mathrm{L}$ & 5,14 & 0,019 \\
\hline * Se calculó mediante la siguiente fórmula, $\mathrm{LLD}=3,3 \mathrm{x}($ Concentración) & xuido/altura \\
del pico & & & & &
\end{tabular}

\section{CONCLUSIONES}

El proceso de extracción, purificación y cuantificacion de ocratoxina A en Coffe arábiga L. "café", de tipo tostado es aceptable aplicando el método de columna de inmunoafinidad y su detección por fluorescencia, corroborado el nivel concentración de 5 ppb. Las concentraciones de las muestras analizadas no superan el límite permitido por los organismos reguladores.

\section{AGRADECIMIENTOS}

Al Vicerrectorado de Investigación y Posgrado de la Universidad Nacional Mayor de San Marcos por el financiamiento, al Instituto de Investigación en Ciencias Farmacéuticas y Recursos Naturales "Juan de Dios Guevara" y al Laboratorio de Investigación y Certificaciones LABICER de la Universidad Nacional de Ingeniería, por el apoyo brindado durante la ejecución de la presente investigación. 


\section{REFERENCIAS BIBLIOGRÁFICAS}

1. Ali R, Ismail M, Bhalli J. Effect of temperature on ochratoxin A production in common cereals by aspergillus species. J Anim Plant Sci. 2013;(23)4: 1316-1320.

2. Revalo A, Ruvio C, Gutiérrez A. La ocratoxina A en alimentos de consumo humano: revisión. Nutr Hosp. 2011; 26(6):1215-1226

3. Binder E, Tan L, Chin L, Handl J, Richard J. Worldwide occurrence of mycotoxins in commodities, feeds and feed ingredients. Anim Feed Sci Tech. 2007;137(3-4):265-282.

4. Organización de las Naciones Unidas para la Agricultura y la Alimentación. Perspectivas a plazo medio de los productos básicos agrícolas. Roma: FAO; 2004.

5. Commission of the European Communities. Regulation (EC) 1881/2006 of 19/12/2006 setting maximum levels for certain contaminants in foodstuffs. Official Journal of European Union. 2006; L364, 5-24.

6. Benites A, Fernandes M, Boleto A, Azevedo S, Silva S, Leitão A. Occurrence of ochratoxin A in roasted coffee samples commercialized in Portugal. Food Control. 2017; 73(2):1223-1228.

7. DIN EN 14132:2009-Foodstuffs: Determination of ochratoxin A in barley and roasted coffee. HPLC method with immunoaffinity column clean-up. EN 14132:2009 Sept. 2009.

8. Neogen. Neocolumn for Ochratoxin WB quantitative test. [Internet][Accesado 15 feb 2020]. Disponible en: https://foodsafety.neogen.com/en/neocolumn-ochratoxin

9. Tafuri A, Ferracane R, Ritieni A. Ochratoxin A in Italian marketed cocoa products. Food Chem. 2014; 88(4); 487-494.

10. Zhang L, Dou X, Zhang C, Logrieco A, Yang M. A Review of Current Methods for Analysis of Mycotoxins in Herbal Medicines. Toxins. 2018;10(2):1-39.

11. Arroyo N, Huertas J, Gamiz L, García A. Control de micotoxinas en alimentos. Granada: Universidad de Granada; 2014 p. 1-16.

12. Moez E, Noel D, Brice S, Benjamin G, Pascaline A, Didier M. Aptamer assisted ultrafiltration cleanup with high performance liquid chromatography-fluorescence detector for the determination of OTA in green coffee. Food Chem. 2019;310(1):1-18.

13. Castellanos O, González O, Guyot B, Fontana T, Guiraud J, Schorr-Galindo S, et al. Efecto de dos técnicas diferentes de tostado en la reducción de ocratoxina A (OTA) en granos de café (Coffea arabica). Control de alimentos. 2011:22(8); 1184-1188.

14. Diana Di, Monbaliu S, Scippo M, Maghuin-Rogister G, Schneider Y, Larondelle Y, Callebaut A, et al. LC-MS/MS multi-analyte method for mycotoxin determination in food supplements. Food Addit Contam. Part A. 2009;26(1):885-895

15. Implvo F, Mendes E, Mbpp O. Quantification of aflatoxins B1, B2, G1, and G2 in pepper by HPLC/fluorescence. J Liq Chromatogr Relat Technol. 2004;27(1):325-334.

16. Monbaliu S, Wu A, Zhang D, Van Peteghem C, De Saeger S. Multimycotoxin UPLC$\mathrm{MS} / \mathrm{MS}$ for tea, herbal infusions and the derived drinkable products. J Agric Food Chem. 2010;58(1):12664-12671.

17. Horwitz W, Alber R. The Horwitz Ratio (HorRat): A Useful Index of Method Performance with Respect to Precision. J AOAC Int. 2006; 89 (1): 1095-1109. 
18. Entwisle AC, Williams AC, Mann PJ, Russell J, Holgura PT, Gilbert J. Combined Phenyl Silane and Immunoaffinity Column Cleanup with Liquid Chromatography for Determination of Ochratoxin A in Roasted Coffee: Collaborative Study. J AOAC Int. 2001; 84(1): 444-450.

19. Pfohl-Leszkowicz, A, Molinié A, Castegnaro, M. Presencia de ocratoxina A, citrinina y fumonisina $\mathrm{B} 1$ en cereales para el desayuno recogidos en el mercado francés. Comparación del análisis de OTA usando o no limpieza de inmunoafinidad antes de HPLC. Revista Mexicana de Micología. 2004;19: 7-15

20. Pfohl-Leszkowicz A, Molinié A, Castegnaro M. Subestimación de fumonisina B1 y ocratoxina A, de matrices complejas mediante el uso de columnas de inmunoafinidad. En micotoxinas y ficotoxinas, Acta del XI Simposio Internacional de la IUPAC sobre micotoxinas y ficotoxinas. 17-21 de mayo de 2004; Bethesda, MD, EE. UU. 